\title{
Neutrino Interactions: Results by Neutrino 2012 and Beyond
}

\author{
Deborah A. Harris \\ Fermi National Accelerator Laboratory, Batavia, Illinois 60510 USA
}

\begin{abstract}
These proceedings will first explain why the fields of both particle and nuclear physics are demanding a better understanding of neutrino interactions. Then they will list the ingredients that go into an effective neutrino interaction program worldwide, and describe how the new and upcoming neutrino interaction experiments and related efforts help to provide those ingredients. In particular, the MINER $\nu \mathrm{A}$ experiment is a dedicated neutrino interaction experiment designed to study neutrino interactions on several different nuclear targets using a fine-grained scintillator detector and is currently taking data at the NuMI beamline at Fermilab. The T2K 280m Off Axis Near Detector at J-PARC, a fine-grained scintillator-based detector which is optimized for studying neutrino interactions on water and scintillator, is also currently taking data. The status of the upcoming $\mathrm{NO} \nu \mathrm{A}$ Near Detector and MicroBooNE experiment will also be described. The proceedings will conclude with a brief discussion of a few ingredients that are not yet in the mix but would make for an even more complete program.
\end{abstract}

Keywords: neutrino interactions, cross-section, nuclear effects

\section{Introduction}

The field of neutrino interactions is seeing an increase in activity, thanks in no small part to the recent advances in our understanding of neutrino mass and mixing. While the original discoveries of neutrino oscillations came from atmospheric and solar neutrinos, the precision measurements of the mixing angles and mass splittings are most easily achieved with a combination of accelerator-based and reactor-based neutrino sources. In order to provide statistics for rare processes at distant detectors, todays neutrino beamlines are extremely intense and allow dedicated neutrino interaction detectors to be relatively modest in size. Furthermore, as the field progresses towards more statistically precise oscillation measurements, the uncertainties in experimental results become more and more dominated by the uncertainties associated with neutrino interactions.

However, the study of neutrino interactions is important even in the absence of neutrino oscillations. There are currently several outstanding mysteries in neutrino cross section measurements, and because of these outstanding mysteries the theoretical picture of the way neutrinos interact in a nucleus is far from complete. One outstanding mystery is the discrepancy between low energy quasi-elastic cross section, as measured by MiniBooNE [1] and SciBooNE [2], and the higher energy quasi-elastic cross section as measured by NOMAD [3].

The nuclear physics community has made a wealth of measurements of charged lepton scattering off of various nuclei in an effort to better understand the nucleus itself [4]. However, these charged lepton measurements are sensitive to the charge weighted quark distributions. By doing similar scattering measurements with neutrinos on a variety of nuclei we can get a better understanding of the valence and sea quark distributions inside the nucleus, and how they are affected by the nuclear environment.

\section{Ingredients}

The first step in achieving a new understanding in the way neutrinos interact is to build much more sensitive detectors than those currently used for oscillation experiments. The MINOS detector, for example, consists of hundreds of $2.5 \mathrm{~cm}$ thick plates of steel each followed by $1 \mathrm{~cm}$ thick scintillator planes

January 12, 2011 
[5]. The transverse and longitudinal granularity of that detector makes it difficult to characterize final state particles and processes, apart from the presence of a final state muon. The Super-Kamiokande detector uses the Cerenkov technique which places a minimum momentum threshold on outgoing particles, which again makes proton and pion final state identification challenging [6].

In order to study neutrino interactions it is also important to have neutrino beams of several energies. As neutrino energies vary from one half to several $\mathrm{GeV}$ even in one neutrino beam, there are several processes that become kinematically possible [7]. Although the quasi-elastic interaction produces a relatively simple final state, even that process seems to be providing mysteries between experiments. Single pion production becomes important at about one $\mathrm{GeV}$ and the deep inelastic scattering process become dominant by about $5 \mathrm{GeV}$. Although a peak neutrino beam energy may be in the quasi-elastic regime, there is always a high energy tail at some level, which will provide feed-down to the signal region through neutral current processes.

The next important ingredient in the study of neutrino interactions is high statistics. The previous most sensitive detectors were bubble chambers which had only handfuls of events. In order to characterize completely a particular neutrino interaction channel, it is not sufficient only to see the final state, but an experiment must explore the full kinematics of that state. For example, the charged current coherent pion production process seemed to be missing from inclusive distributions of pion plus muon final states in MiniBooNE [8] and SciBooNE [9]. However, at NuInt09 ScibooNE showed that for certain angular regions there seemed to be a coherent component, while for others it was absent [10]. The neutral analog of this process is a rare but easily accepted background to electron neutrino appearance analyses, so the process overall needs to be well understood. Also, these coherent processes are expected to have similar cross sections between neutrinos and antineutrinos, and as such will have an even more uncertain background contribution in antineutrino mode.

Another crucial ingredient for neutrino interaction experiments is a well-understood flux. For example, the current discrepancy between the low energy and high energy quasi-elastic cross section measurements described above might simply be a few sigma shift in the flux uncertainties of one of the experiments.
Finally, another important ingredient in future interaction measurements is that they be done on a broad range of nuclear targets. Although oscillation experiments will naturally want to study neutrino interactions on the target nuclei that are present in the far detector, coming up with a consistent model of how the nucleus affects neutrino interactions is only possible when a broad range of nuclei is studied (see reference [11] for a comparison of target nuclei and energies of oscillation experiments and scattering experiments). The nuclear physics community has a wealth of charged lepton scattering data on elements as light as Hydrogen [12], so for neutrino scattering experiments, the lower the atomic number of the target nuclei the better.

\section{Ingredients in Current Generation of Ex- periments}

\subsection{Detectors}

All of the next generation neutrino interaction experiments take advantage of extremely finegrained tracking volumes, using a variety of different technologies.

The MINER $\nu \mathrm{A}$ detector is made of finely segmented scintillator planes that are read out by wavelength shifting fibers [13]. The planes themselves are comprised of nested triangular-shaped scintillator bars, where the base of each triangle is $3.3 \mathrm{~cm}$ and the height is $1.7 \mathrm{~cm}$. By comparing light from neighboring bars a position resolution of better than $3.5 \mathrm{~mm}$ can be achieved. The light from the fibers is sent through clear fiber cables to Hamamatsu multi-anode photo multiplier tubes, which in turn are read out by front end electronics that use Trip-t chips that were originally developed by DZero. The scintillator planes have collars of lead around their edges to provide side electromagnetic calorimetry. The outer hadron calorimetry is provided by steel hexagons that surround the inner tracker region that are instrumented with scintillator bars. The entire detector sits in front of the MINOS Near Detector which serves as a muon spectrometer. The granularity allows high resolution on individual neutrino interactions, and secondary (detached) vertices are visible (as shown in the data events shown in figure 1, as are multi-particle final states, and particle identification using energy deposition along tracks that range out in the detector.

The T2K near detector complex consists of several detectors: there are two in particular that will 

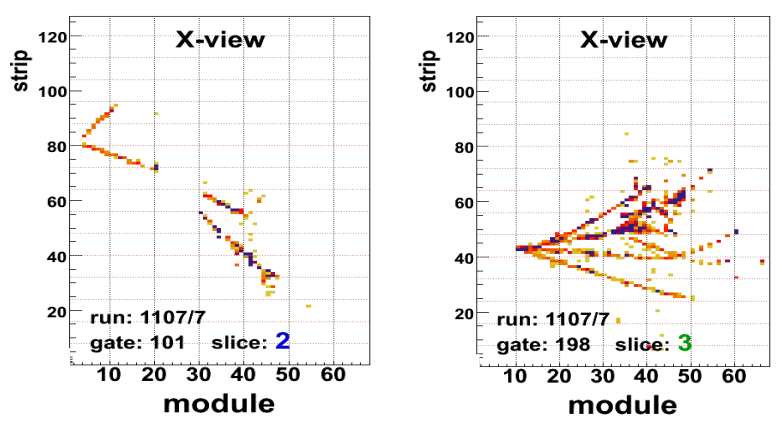

Figure 1: Two different $\mathrm{x}$ views of event displays from the MINER $\nu \mathrm{A}$ detector. These events came from a time when over half of the detector was installed and reading out events in an antineutrino beam

be used for neutrino interaction measurements [14]. The most upstream detector, the P0D, is optimized to study electromagnetic final states in both water and scintillator. The active material is scintillator, using triangular-shaped bars with the same cross section as those in the MINER $\nu \mathrm{A}$ inner detector. The scintillator planes are interspersed with planes containing water, so that a direct comparison can be made between interactions on carbon and interactions on water. There is also lead and brass as part of the P0D. The fiducial mass of carbon (oxygen) is $1.8(0.9)$ tons. The scintillator is read out with wavelength shifting fibers and silicon photomultipliers.

Downstream of the P0D are three time projection chambers, the first two of which are followed by an additional fine grained detector (FGD) that contains both scintillator planes and space for water. This detector suite is designed to measure exclusive final states. The interactions on water will be determined by subtraction (where the first FGD does not contain water and the second one does). Each fine grained tracker has a fiducial mass of 1.3 tons. Figure 2 shows an event display from the T2K early running with a neutrino interaction in the P0D that has final state particles extending into the downstream fine grained detectors and TPC's.

Finally, at the downstream end of the T2K $280 \mathrm{~m}$ detector there is an electromagnetic calorimeter to ensure containment. There is also side calorimetry around both the P0D and the TPC's and FGD's to catch particles that are exiting the side of the

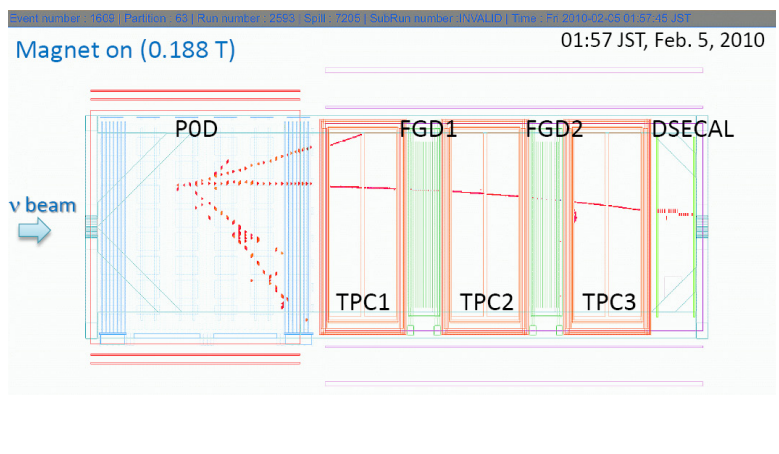

Figure 2: A neutrino interaction in T2K's Near Detector complex: the event originates in the P0D and the final state particles cross to the TPC's and the downstream electromagnetic calorimeter.

tracking volume. The entire detector is in a magnetic field of about $0.188 \mathrm{~T}$. With the combination of the TPCs and the magnetic field the detector has good capability of complete particle identification even for multi-track events.

The NO $\nu \mathrm{A}$ near detector consists of extrusions filled with liquid scintillator, similar to the far detector [15]. The extrusion cross-section is $3.87 \mathrm{~cm}$ by $6 \mathrm{~cm}$, and the horizontal (vertical) extrusions are $3 \mathrm{~m}(4.5 \mathrm{~m})$ long. Each extrusion contains both the liquid scintillator and a wavelength shifting fiber which is read out using an avalanche photo-diode (APD). Since the peak neutrino energy for $\mathrm{NO} \nu \mathrm{A}$ is $2 \mathrm{GeV}$, there is a $1.7 \mathrm{~m}$ long muon range stack at the downstream end of the $\mathrm{NO} \nu \mathrm{A}$ near detector. The stack is comprised of ten planes of steel interspersed with 10 planes of extrusions identical to the rest of the detector. The entire detector is $16 \mathrm{~m}$ long so the electromagnetic component of the events in the fiducial volume of the experiment will be well-contained. The total mass of the $\mathrm{NO} \nu \mathrm{A}$ near detector is 209 (126) total (active) tons. The expectation is that the fiducial mass of the detector will be 23 tons.

The MicroBooNE detector consists of a liquid argon time projection chamber, with 150 (89) tons total (active) of liquid argon [16]. Because argon also scintillates, there will be 30 PMTs to collect that scintillation light for use in event triggering. The time projection chamber itself is $2.5 \mathrm{mx} 2.5 \mathrm{mx} 10.4 \mathrm{~m}$ long, and the wire pitch will be $3 \mathrm{~mm}$. Given the small wire pitch and readout speed, the segmen- 
tation for MicroBooNE will be significantly higher than that of other neutrino detectors. The purity requirements of this detector mean however that the cross section measurements will be strictly for argon target nuclei. The granularity of this detector surpasses that of the other detectors described here, though, so much more information per event is able to be collected and available for use in reconstruction.

\subsection{Beamlines}

The next generation of neutrino interaction experiments will span the interesting region of neutrino energies across the threshold of non-quasielastic processes. The T2K $280 \mathrm{~m}$ fine grained near detector complex sits off axis, and has a peak neutrino energy of about $600 \mathrm{MeV}$ in the current beamline configuration. This peak is relatively narrow and the high energy tail is extremely low [17]. The MicroBooNE detector will sit in the Booster Neutrino Beam, and in that location will see a broad band of neutrino energies that is centered on about $1 \mathrm{GeV}[18]$. Because it is on axis and is made with $8 \mathrm{GeV}$ protons, there will be a small high energy tail at the few per cent level.

The NO $\nu \mathrm{A}$ near detector will operate in two locations: the detector will be constructed and commissioned above ground in a special building that is $107 \mathrm{mrad}$ off the NuMI beamline axis, and in that location it will see a low intensity neutrino beam which has a relatively high electron neutrino content, and also a $2 \mathrm{GeV}$ muon neutrino flux that comes from kaon decays [15]. By the time the $\mathrm{NO} \nu \mathrm{A}$ far detector is operational at full mass, the $\mathrm{NO} \nu \mathrm{A}$ near detector will be located in a new alcove underground, at a location very much closer to the $14 \mathrm{mrad}$ off the NuMI axis, similar to the off axis angle of the far detector. In both $\mathrm{NO} \nu \mathrm{A}$ locations, since the detector is off axis there will be a very small high energy tail.

The MINER $\nu$ A detector sits on axis in the NuMI beamline, and in that location can see a variety of neutrino fluxes, depending on the configuration of the upstream beamline focusing system. MINER $\nu \mathrm{A}$ will run in both a low energy configuration where the peak neutrino event energy is about $3.5 \mathrm{GeV}[20]$, and after 2013 when the $\mathrm{NO} \nu \mathrm{A}$ experiment is running, MINER $\nu \mathrm{A}$ will run in a medium energy configuration where the peak neutrino event energy is around $7 \mathrm{GeV}$. Since the detector is on the main axis of the beamline, and because the incident proton energy is $120 \mathrm{GeV}$, there is a significant high energy tail that will be present in all the MINER $\nu \mathrm{A}$ running configurations [19].

\subsection{Statistics}

Since the MINER $\nu \mathrm{A}$ experiment is a dedicated on axis cross section experiment with the highest energy beam, it is slated to have the highest statistics, thereby alllowing it to probe thoroughly the details of neutrino interactions in the few $\mathrm{GeV}$ energy range. With an exposure of $4 \times 10^{20}$ protons on target in the standard low energy configuration, and $12 \times 10^{20}$ protons on target in the medium energy configuration, there will be some 9 million charged current neutrino events on the 3 ton fiducial mass of plastic scintillator active target. Of those, 4.3 million of them are deep inelastic scattering events, 1.7 million are in the resonance region, and 0.8 million of them are quasi-elastic events.

The T2K 280m near detector complex will expect some 300 (150) thousand quasi-elastic events in the carbon (oxygen). Similarly, the POD should be able to collect some 26 (14) thousand neutral pion events in the carbon (oxygen). These statistics are for an assumed exposure of $5 \times 10^{21}$ protons on target [14]

The NO $\nu \mathrm{A}$ near detector is expected to collect some ten thousand charged current events while it is located on the surface at $107 \mathrm{mrad}$ from the NuMI beamline, and then another 1.7 million charged current events for a 20 ton fiducial mass and one year of running $\left(6 \times 10^{20}\right.$ protons on target $)$. Of those 1.7 million events, 440 thousand will be quasi-elastic events, and 578 thousand will be deep inelastic scattering events [15].

MicroBooNE is expected to collect a total of 84 thousand charged current events for an exposure of $6 \times 10^{20}$ protons on target in the Booster beamline. Of those events, some 53 thousand of them are expected to be quasi-elastic events [16].

\subsection{Flux Determination}

A challenge to all neutrino interaction measurements is how to determine the flux. For high energy neutrino beams, there is a low hadron energy method which relies on the fact that for deep inelastic scattering process, the low $y$ cross section as a function of neutrino energy approaches a constant as $y$, the inelasticity of the interaction, approaches zero. So if the experiment is dominated by DIS events, and iif one can cleanly separate events with little or no hadron energy, the flux can be determined. However, for the current and future oscillation experiments, the beamline is operating where 
a large fraction of events are not deep inelastic scattering, and so this technique cannot be applied for a robust low energy flux measurement.

One way that neutrino experiments try to understand their fluxes is by incorporating results from hadron production experiments, and these are discussed elsewhere in these proceedings [21]. This article will focus specifically on in situ techniques to determine the flux.

The MINER $\nu \mathrm{A}$ experiment will make use of the flexibility of the NuMI beamline to take special runs in several different configurations to test its flux model[22]. These different configurations are achieved by moving the target relative to the focusing horn system, and by changing the horn current. By making these two sets of changes the experiment can sweep through the transverse and longitudinal momentum spectrum respectively of the produced pions. One technique involves taking neutrino data at each of these different configurations for a limited amount of time, and another technique involves measuring the muon rates after several different amounts of shielding, again for many different horn currents and for several different target positions. Finally, the MIPP experiment at Fermilab [23] took hadron production data on an earlier NuMI target, and the forthcoming results can also be used to help constrain the flux model for $\mathrm{MINER} \nu \mathrm{A}$.

The T2K 280m Near Detector complex includes not only the two detectors described above, but also a large array of steel-scintillator modules that are arranged in a cross to measure the neutrino beam center and width. This is one way to constrain the flux model, in addition to the muon flux measurements that are planned with the T2K muon monitor system [14]. Finally, there is also a hadron production experiment, NA61 at CERN, that measured hadron production using a T2K target replica [24].

\subsection{Nuclear Targets}

As a dedicated neutrino interaction experiment with a large nuclear physics contingent on the collaboration, MINER $\nu \mathrm{A}$ is studying the largest range of target nuclei. The experiment has already installed thin targets of lead, carbon, and steel (one of which is shown in Figure 3) in addition to the active scintillator target. The experiment also has plans to install both a water target and a liquid helium target. The T2K 280ND complex has brass, lead, scintillator, and water in the neutrino detectors, and the design is such that cross sections on

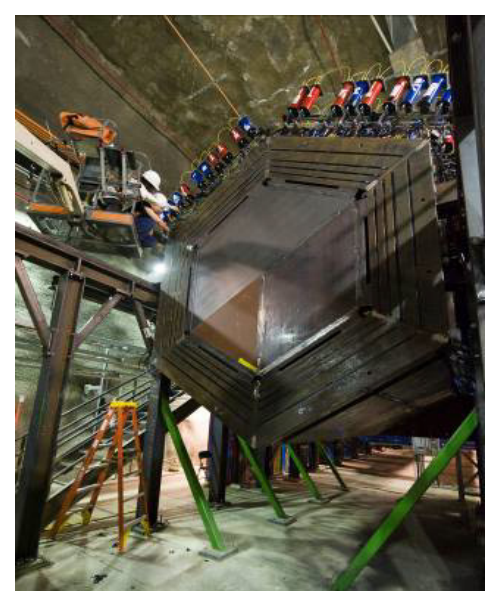

Figure 3: Photo of one of the MINERvA nuclear targets, showing graphite, iron, and lead wedges which will be surrounded by active scintillator planes

carbon and oxygen can be determined by subtraction techniques. $\mathrm{NO} \nu \mathrm{A}$ and MicroBooNE will only be producing measurements on single nuclei species (carbon and argon, respectively) but combined with the information that comes from the broad range of nuclear targets in MINER $\nu \mathrm{A}$ the single-target experiments may still be able to provide input on nuclear effects.

\section{Current Status of Data Taking}

The MINER $\nu \mathrm{A}$ experiment started accumulating physics-quality antineutrino data with a partially complete detector in November 2009, and finished the detector assembly and commissioning in March 2010. By that point the experiment had integrated about $0.84 \times 10^{20}$ protons on target in antineutrino mode. On March 22, 2010 the NuMI beamline was configured to provide a Low Energy neutrino beam, and MINER $\nu \mathrm{A}$ officially started its run. At the time of the Neutrino 2010 conference the MINER $\nu \mathrm{A}$ experiment had accumulated over $0.8 \times 10^{20}$ protons on target in neutrino mode, or about $20 \%$ of its nominal low energy running. The detector livetimes quickly reached above $95 \%$, and have remained high since then. The detector tracking is already at the $3.2 \mathrm{~mm}$ transverse coordinate resolution at the current stage of data analysis. The light yield of the scintillator is good enough that particles can be identified based on the energy deposited at the end of a stopping particle track. Figure ?? shows one of three views from two different event displays during the early antineutrino run. 
The T2K 280m Near Detector complex saw its first neutrino events on January 23, 2010, and between that date and May 12010 , the T2K beamline has integrated $0.234 \times 10^{20}$ protons on target. This is roughly about 2

The Time Project Chamber tracking performance is already yielding a transverse position resolution of $0.6 \mathrm{~mm}$. The ability to do particle identification with the TPC has also been demonstrated by comparisons of the reconstructed momentum versus the energy loss for both positive and negative particle samples.

\section{Results by Neutrino 2012}

In the next two years, MINER $\nu$ A plans to take up to $4.9 \times 10^{20}$ protons on target worth of low energy neutrinos. This beam is well-suited to study exclusive channels because of the low event rate and the low multiplicity in the events at the peak energy, compared to the medium energy running that will be concurrent with the $\mathrm{NO} \nu \mathrm{A}$ experiment run. Of the $4.9 \times 10^{20}$ protons on target, $0.9 \times 10^{20}$ of them are slated for special runs in altered neutrino beam configurations. These special runs are designed to reduce the flux uncertainties on the low energy neutrino beam by testing the underlying hadron production model for different focusing geometries. Finally, by comparing exclusive interactions off different target nuclei, MINER $\nu \mathrm{A}$ will be able to probe nuclear effects of neutrino interactions and look for process-dependent effects.

The T2K 280m Near Detector complex plans to take data at the level of approximately 1021 protons on target per year by the year 2012. The main foci of the T2K Near Detector analysis in these early years are the quasi-elastic cross section, which comprises the signal process, and the neutral pion production which is the most important background to the electron neutrino appearance search at T2K.

By the year 2012 the $\mathrm{NO} \nu \mathrm{A}$ near detector will have been operating at about $110 \mathrm{mrad}$ from the NuMI beamline. With an exposure of $2 \times 10^{20}$ protons on target in the neutrino configuration, $\mathrm{NO} \nu \mathrm{A}$ will be able to collect about ten thousand events to benchmark the $\mathrm{NO} \nu \mathrm{A}$ detector performance. 2012 is also the year that the current schedule calls for the $\mathrm{NO} \nu \mathrm{A}$ near detector underground excavation to begin.

By the year 2012 the MicroBooNE experiment should be assembling and installing their time projection chamber in the cryogenic vessel. The cur- rent schedule calls for filling the vessel with Argon by February 2013 [24].

\section{Missing Ingredients}

Much progress is being made in the field of neutrino interactions, and we will know a lot more in the next two years than we have known in the past few decades about neutrino interactions. However, there are still a few ingredients that are not yet part of the approved plan but which would significantly extend the program.

One such ingredient is a fine-grained neutrino detector sitting in a $2 \mathrm{GeV}$ neutrino beam, which is the peak energy at the $\mathrm{NO} \nu \mathrm{A}$ far detector location. Although the $\mathrm{NO} \nu \mathrm{A}$ detector itself is far more finegrained than for example the MINOS detector, it is still about a factor of 10 more coarse grained than the MINER $\nu \mathrm{A}$ detector. Although the MINER $\nu \mathrm{A}$ experiment will see significant flux at $2 \mathrm{GeV}$ during the low energy running, the majority of events are from higher energies and the feed down to those energies in neutral current interactions, will be challenging to untangle. There is currently a proposal to put a 10 ton detector that is made of SciBar-like scintillator bars in front of the $\mathrm{NO} \nu \mathrm{A}$ near detector. This proposal is called $\mathrm{SciNO} \nu \mathrm{A}$ and was presented to the Fermilab Physics Advisory Committee in the fall of 2010 [24]. SciNO $\nu$ A would be able to collect about a million events in a 1 year run during the $\mathrm{NO} \nu \mathrm{A}$ era when the NuMI beamline will be integrating some $6 \times 10^{20}$ protons on target per year.

Another ingredient which is technically more challenging but would extend the field even further is a high statistics suite of measurements of neutrino interactions on hydrogen and deuterium. Many anomalies in neutrino interaction measurements are interpreted as a lack of understanding of the nuclear environment. A survey of interactions on these lightest nuclei would enable definitive measurements of the proton and neutrons quasi-elastic form factors. Furthermore, because of the following definitions of the $F_{2}$ structure function on the proton,

$$
\begin{aligned}
& F_{2}^{\nu p}=2 x(d+\bar{u}+s) \\
& F_{2}^{\overline{\nu p}}=2 x(\bar{d}+u+\bar{s})
\end{aligned}
$$

a high statistics set of measurements of neutrino and antineutrino scattering on protons would yield a measurement of the ratio of down to up quarks in the proton, for those quarks that have a high 
fraction of the protons momentum (in other words, those at high $x$ ). This would have implications not only for the structure of the nucleon, but for hadron collider measurements as well.

\section{Conclusions}

A detailed study of neutrino interactions is in higher demand than ever before because of the need to measure neutrino interactions, and it is no coincidence that the facilities to do those studies are also available at an unprecedented level. The next few years will see the commissioning and operations of a series of fine-grained detectors that have the potential to revolutionalize our understanding of both the neutrino and the nucleon. There are several neutrino energies that will be available worldwide, offering a chance to study the entire span between a quasi-elastic dominated regime all the way up to where most interactions come from Deep Inelastic Scatters. Between the planned hadron production experiments and in situ measurements of neutrino event rates for several different fluxes, the field can develop techniques for new levels of precision in flux predictions. The broad range of nuclear targets that are available at the MINER $\nu \mathrm{A}$ detector will provide stringent tests of the models of nuclear dynamics and will permit side by side comparisons of how the nucleus affects the way we measure neutrinos. There is still a lot to be done as we figure out a way to ultimately measure with high statistics neutrino scattering on hydrogen and deuterium.

\section{References}

[1] A. A. Aguilar-Arevalo et al., [MiniBooNE Collaboration] Phys. Rev. D81: 092005, 2010. arXiv: 1002.2680 [hep-ex]

[2] Jose Luis Alcaraz-Aunion et al.,[SciBooNE Collaboration], NUINT 2009 Proceedings, AIP Conf. Proc. 1189:145-150, 2009. arXiv:0909.5647 [hep-ex]

[3] $\mathrm{V}$ Lyubushkin et al., [NOMAD Collaboration] Eur. Phys. J. C63: 355-381, 2009. arXiv:0812.4543 [hep-ex]

[4] X. Qian, T. Horn, B. Clasie, et al., Phys. Rev. C81: $055209,2010$.

[5] D. G. Michaels et al., [MINOS Collaboration] Nucl. Instrum. Meth. A596: 190-228, 2008.

[6] Y. Fukuda et al., Nucl. Instrum. Meth. A501, 418 (2003).

[7] K.S. McFarland, Neutrino Interactions, Published in Neutrinos in Particle Physics, Astrophysics and Cosmology, October 2008. arXiv:0804.3899 [hep-ex]

[8] J. Wilking for MiniBooNE Collaboration, NuInt 2009 Proceedings, AIP Conf. Proc. 1189:236-242,2009.
[9] K. Hiraide et al., [SciBooNE Collaboration] Phys. Rev. D78: 112004, 2008.

[10] Y. Kurimoto et al., [SciBooNE Collaboration] Phys. Rev. D81:111102,2010.

[11] D. Schmitz, Review of Current and Future Neutrino Cross-Section Experiments, FERMILAB-CONF09-711-E, July 2009, NuFact09 Conference Proceedings AIP Conf. Proc. 1222:52-56,2010.

[12] M.E. Christy and P. Bosted, Phys. Rev C81: 055213, 2010 .

[13] B. Eberly for the MINER $\nu$ A Collaboration, Status of the MINER $\nu$ A Experiment NuFact09 Conference Proceedings, July 2009, AIP Conf. Proc. 1222: 253-256, 2010

[14] S. Boyd, WIN 09 presentation http://indico.Cern.ch/contributionDisplay.py? contribID=167\&sessionID=18\&conf $i d=54503$.

[15] K. Heller, these proceedings

[16] M. Soderberg, these proceedings

[17] T. Kobayashi, these proceedings

[18] A. A. Aguilar-Arevalo et al., [MiniBooNE Collaboration] Phys. Rev. D79, 072002 (2009)

[19] D.S. Ayres et al., [NO $\nu \mathrm{A}$ Collaboration], The $\mathrm{NO} \nu \mathrm{A}$ Technical Design Report, FERMILAB-DESIGN-200701, October 2007

[20] Kopp, S., FERMILAB-CONF-05-093-AD, Proceedings of PAC 05, arxiv: [physics] 0508001

[21] A. Blondel, these procedings

[22] S. Kopp, these proceedings

[23] T.S. Nigmanov et al., [MIPP Collaboration] Nucl. Instrum. Meth. A598: 394-399,2009. arXiv:0903.2463 [physics.ins-det], and N. Graf et al., [MIPP Collaboration] Nucl. Instrum. Meth. A615: 27-32, 2010. arXiv:0909.0971 [hep-ex]

[24] N. Abgrall et al, [on behalf of T2K and NA61 Collaborations] NA61-SHINE: Hadron Production Measurements for Cosmic Ray and Neutrino Experiments arXiv:1005.3692

[25] C. James, private communication

[26] J. Paley et al., SciNO $\nu \mathrm{A}$ : A Measurement of Neutrino-Nucleus Scattering in a Narrow-Band Beam, FERMILAB-PROPOSAL-1003, October 2010 